\title{
Systemic inflammation and sepsis. Part I: Storm formation
}

\author{
Juan B. Dartiguelongue, M.D..$^{a, b, c}$
}

\begin{abstract}
Sepsis is still a major cause of morbidity and mortality. It results from a dysregulated immune response to infection that leads to organ and system dysfunction.

The inflammatory response to pathogenic microorganisms implies a dynamic, complex chain of eventsleading toendothelial and immune system activation. The purpose of this process is to control infection and repair tissues. However, both host and microorganism factors may result in severe forms of systemic inflammation with a high mortality rate. Sepsis falls within this complexscenario, where the inflammatory storm and the causative microorganism converge in a severe multisystem presentation.

This manuscript is divided into two parts. Part I describes the mechanisms triggering systemic inflammation and progression to sepsis, together with its main biological markers. Part II analyzes the mechanisms leading to organ dysfunction. Keywords: sepsis, septicshock, systemicinflammatory response syndrome, cytokines.
\end{abstract}

http:/ / dx.doi.org/10.5546/ aap.2020.eng.e527

To cite: Dartiguelongue JB. Systemic inflammation and sepsis. Part I: Storm formation. Arch Argent Pediatr 2020;118(6):e527-e535.

\section{GLOSSARY}

ATP: adenosine triphosphate.

CRP: C-reactive protein.

DAMPs: damage-associated

molecular patterns.

DNA: deoxyribonucleic acid.

HSPs: heat shock proteins.

ICAM-1: intercellular adhesion molecule-1.

IFN- $\gamma$ : interferon gamma.

IL-1: interleukin 1.

IL-10: interleukin 10.

IL-12: interleukin 12.

IL-15: interleukin 15.

IL-18: interleukin 18.

IL-2: interleukin 2.

IL-4: interleukin 4.

IL-6: interleukin 6.

IL-7: interleukin 7.

IL-8: interleukin 8.

LBP: lipopolysaccharide-binding protein.

MBL: mannose-binding lectin.

PAMPs: pathogen-associated molecular patterns.

PRRs: pattern recognition receptors. SIRS: systemic inflammatory

response syndrome.

SNP: single nucleotide

polymorphism.

TGF- $\beta$ : transforming growth factor beta.

TLRs: Toll-like receptors.

TNF- $\alpha$ : tumor necrosis factor alpha.

VCAM-1: vascular cell adhesion molecule-1.

\section{INTRODUCTION}

Currently, sepsis is defined as a life-threatening severe organ dysfunction caused by a dysregulated immune response to infection. ${ }^{1}$ It is still one of the main causes of morbidity and mortality, and depends on the patient's age, environment (community, hospitalization wards, intensive 
care units), immune system maturity, and the microorganism involved. ${ }^{2}$ The rate of sepsis is very high among newborn infants and critically ill and immunocompromised patients. ${ }^{3}$

Progression to sepsis implies a dynamic, complex series of events leading to endothelial and immune system activation. Such inflammatory, self-sustaining, dysregulated response results in severe systemic inflammation. ${ }^{4}$ The consequences of this process, involving both host and microorganism factors, include endothelial damage, microvascular dysfunction, alterations in tissue oxygenation, and, finally, multiple organ dysfunction. ${ }^{5,6}$

Objective: To describe the mechanisms leading to systemic inflammation and progression to sepsis, together with its main biological markers.

\section{INNATE IMMUNITY AND INFLAMMATION}

\section{Cell activation and inflammatory response}

The innate immune response, a very ancient one from a phylogenetic perspective, brings a set of cellular and humoral effectors into play that trigger an immediate immune response and, if necessary, initiate the involvement of specific immune effectors ( $B$ and $T$ cells), so that all together can eradicate or control the infection from the beginning. This response is initiated by pattern recognition receptors (PRRs), located on the cell membrane (as well as on intracellular organelles) of monocytes, macrophages, and neutrophils. These receptors recognize specific molecular structures typical of microorganisms. The most widespread PRRs include Toll-like receptors (TLRs) and others known as nucleotidebinding oligomerization domain (NOD) and retinoic acid-inducible gene 1 (RIG-1). ${ }^{7-9}$

Bacterial patterns recognized by these receptors are known as pathogen-associated molecular patterns (PAMPs); the most widespread PAMPs include Gram-negative bacterial lipopolysaccharides and peptidoglycans and lipoteichoic acid in Gram-positive bacteria. PAMPs are recognized by PRRs, therefore triggering the inflammatory process. ${ }^{10,11}$ Specifically, lipopolysaccharides and peptidoglycans bind to TLR-4 and TLR-2, respectively. Such binding initiates an intracellular signaling pathway

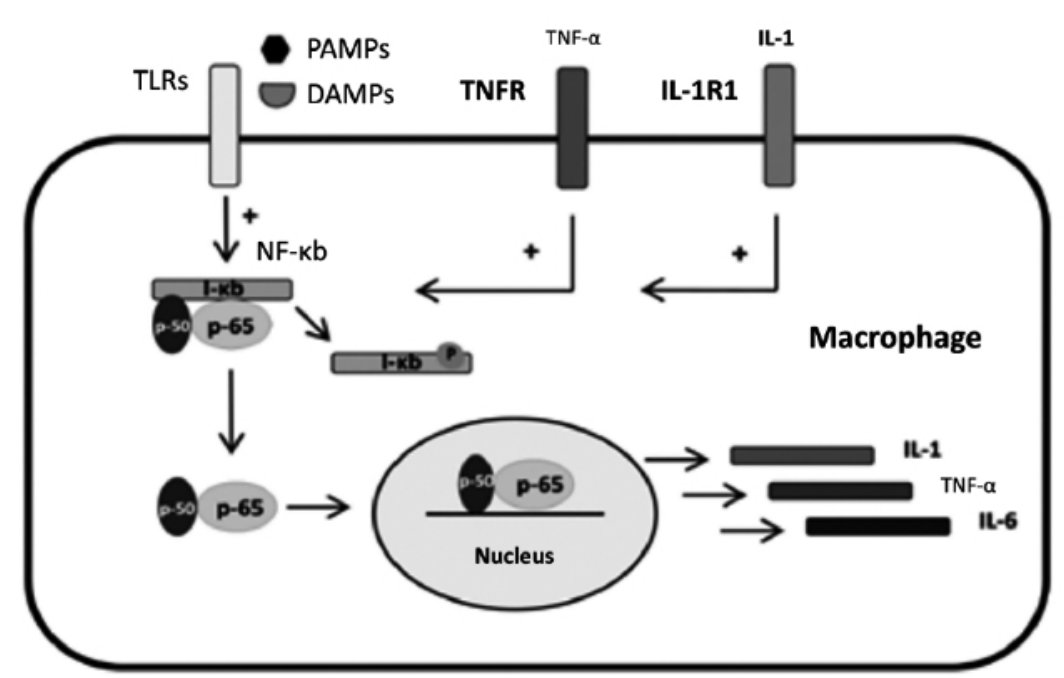

Representation of macrophage activation through the interaction between PAMPs/DAMPs and TLRs and TNF- $\alpha$ and IL-1 action on their receptors (TNFR and IL-1R1, respectively). With quiescent cells, NF- $k b$ is found in cytoplasm and forms a heterodimer made up of subunits p-50 and p-65, bound to an inhibitory protein known as I- $\kappa b$. The activation of TLRs and of TNF- $\alpha$ and IL-1 receptors, among others, triggers the activation of the I- $\kappa \mathrm{b}$ kinase complex, responsible for the phosphorylation that leads to the proteolytic degradation of I- $k \mathrm{~b}$. This allows the translocation of the $\mathrm{p}-50 / \mathrm{p}-65$ heterodimer to the nucleus, where it binds to $\mathrm{kb}$ sequences in specific gene promoter regions.

TLRs: Toll-like receptors; NF-kb: transcription nuclear factor; PAMPs: pathogen-associated molecular patterns;

DAMPs: damage-associated molecular patterns; TNF- $\alpha$ : tumor necrosis factor alpha; IL-1: interleukin 1; IL-6: interleukin 6.

Source: Developed by the author. 
that implies the nuclear translocation of the transcription factor kappa-b (NF- $k b)$ and the subsequent cell activation and expression of genes involved in inflammatory response (Figure 1). These genes include that of interleukin 1 (IL-1), interleukin 6 (IL-6), tumor necrosis factor alpha (TNF- $\alpha$ ), several chemokines (intercellular adhesion molecule-1 [ICAM-1], vascular cell adhesion molecule-1 [VCAM-1]), and nitric oxide synthase enzyme, among others.

In turn, PRRs recognize and are activated by circulating molecules known as damageassociated molecular patterns (DAMPs), released during inflammatory aggression. These are nuclear, cytoplasmic, and mitochondrial structures that acquire new properties once in the extracellular medium. Examples include mitochondrial deoxyribonucleic acid (DNA), adenosine triphosphate (ATP), heat shock proteins (HSPs), high mobility group box 1 (HMGB-1) proteins, and S100 proteins (soluble in $100 \%) .{ }^{12}$

The vascular endothelium also includes TLRs that recognize lipopolysaccharides and other microbial products. In addition, it expresses TNF- $\alpha$ and IL-1 receptors, several cytokines, and complement activation products (C3a, $\mathrm{C} 5 \mathrm{a}$ ). Such interactions cause endothelial activation, which translates physiologically into vasodilation, increased vascular permeability and adhesion molecule expression (selectins, ICAM-1, VCAM-1, etc.) that regulate leukocyte traffic (Figure 2). The resulting hyperemia, together with a change in vascular permeability and activated polymorphonuclear cell invasion, supports the cardinal signs of inflammation.

The TNF- $\alpha$ produced by activated macrophages and neutrophils stimulates its own release (autocrine action) and that of other cytokines and inflammatory mediators (Figure 1), both by the cell itself and circulating macrophages, neutrophils, and endothelial cells (paracrine action). These mediators include IL-1, interleukin 2 (IL-2), IL-6, interleukin 8 (IL-8), and interleukin 10 (IL-10), platelet-activating factor, interferons, and eicosanoids. ${ }^{7,8}$ In turn, polymorphonuclear cell activation implies the expression of adhesion molecules to the activated endothelium (recruitment), the generation of reactive oxygen intermediates, increased phagocytic capacity, lysosomal enzyme release, and increased class I and II human leukocyte antigens (HLAs) activity and expression.

The purpose of all the mechanisms set in motion during this process is infection control and tissue repair. They are mainly regulated based on pro-inflammatory and anti-inflammatory cytokine development. ${ }^{10}$ Such balance modulates leukocyte and endothelial activation, thus limiting the inflammatory process at the injury site.

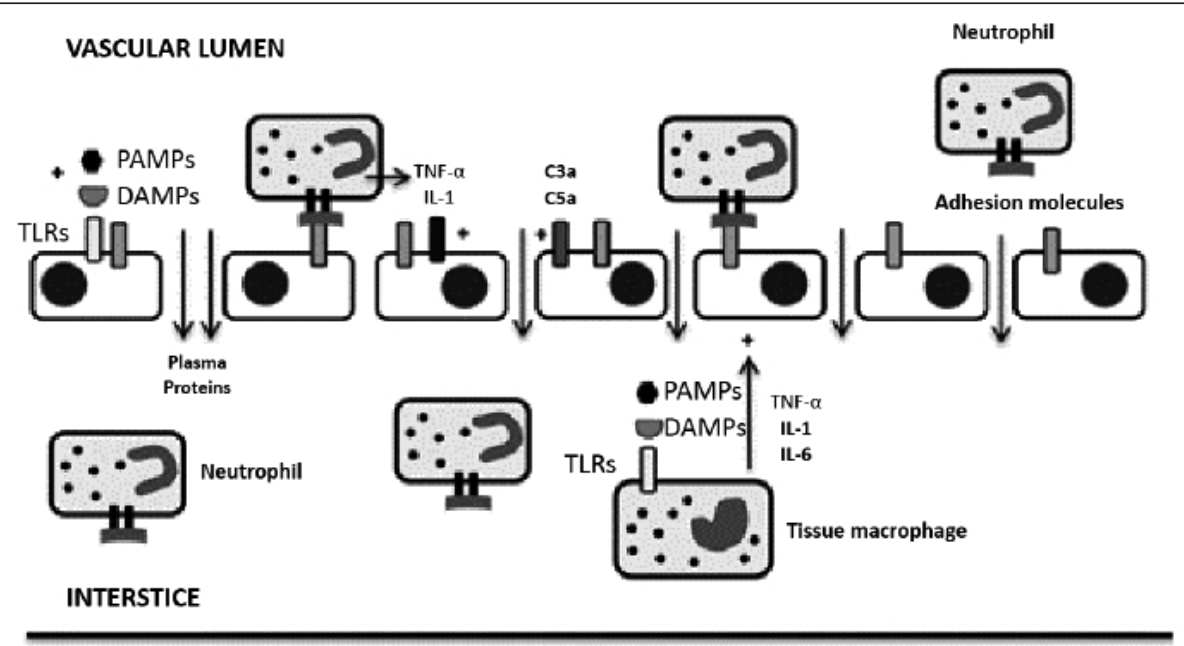

Representation of endothelial activation mediated by PAMPs, DAMPs, cytokines, and complement activation products. The coordinated action of these mediators causes vasodilation, hyperemia, increased vascular permeability, and adhesion molecule expression regulating leukocyte traffic.

PAMPs: pathogen-associated molecular patterns; DAMPs: damage-associated molecular patterns;

TNF- $\alpha$ : tumor necrosis factor alpha; IL-1: interleukin 1; IL-6: interleukin 6.

Source: Developed by the author. 


\section{Cytokines}

Macrophage and neutrophil activation through TLRs triggers a gene transcription process involving the genes of different cytokines. Some of these induce a local and systemic inflammatory response (TNF- $\alpha$, IL-1, IL-6); others guide the future course of immune adaptive response (interleukin 12 [IL12], interleukin 18 [IL-18]), mediate leukocyte recruitment to the inflamed tissue (IL-8 and several chemokines), and induce leukocyte precursor cell proliferation and/or differentiation by acting at the level of the bone marrow (interleukin 7 [IL-7], interleukin 15 [IL-15]).12,13

Both TNF- $\alpha$ and IL-1 and IL-6 play a key role in the initial stages of the inflammatory process thanks to the mediation of a set of activities tending to the development of systemic inflammation..$^{12-16}$ The actions common to several cytokines are detailed in Table 1.

Some cytokines, known as anti-inflammatory cytokines, inhibit TNF- $\alpha$ and IL-1 production. However, their effects are not universally antitransforming growth factor beta (TGF- $\beta$ ), and IL-10. While they stimulate the immune system, improve B cell function (immunoglobulin proliferation and release), and promote cytotoxic T cell development, they inhibit the production of other cytokines by the mononuclear system and monocyte-dependent T-helper cells.

\section{Complement system}

This is one of the humoral mechanisms typical of innate immunity. It is a proteolytic cascade of successive activation that goes from an inactive state to an active state through the proteolysis of its components. ${ }^{18,19}$ Its biological functions include causing an inflammatory response (C3a,
$\mathrm{C} 5 a)$, opsonizing microorganisms (C3b), directly lysing bacteria (C5-C9), and promoting a type-B response ( $\mathrm{C} 3 \mathrm{~b}$ degradation products). ${ }^{18}$

Complement activation (Figure 3 ) is triggered by structures present on microorganism surface (alternative pathway), immune complexes (classical pathway) or mannose-binding lectin (MBL) in what is known as the lectin pathway.

Inflammatory activity is mediated by the $\mathrm{C} 3 \mathrm{a}$ and $\mathrm{C} 5 \mathrm{a}$ components. They both induce neutrophil and monocyte chemotaxis, recruitment, and activation. In turn, they have a pronounced anaphylactic activity leading to mast cell degranulation, ${ }^{18-21}$ and the subsequent release of vasoactive amines (histamine and serotonin), leukotrienes, chemokines, and cytokines (TNF- $\alpha$, interleukin 4 [IL-4]). This favors local blood flow and vascular permeability. In addition, $\mathrm{C} 3 \mathrm{a}$ and C5a directly activate the endothelium, which mediates an increased permeability and adhesion molecule expression. They also stimulate platelet activation. ${ }^{20,21}$

\section{Inflammatory amplification}

Sometimes, the release of inflammatory mediators exceeds the boundaries of the infection site. This leads to an amplified, dysregulated systemic inflammatory response involving otherwise healthy tissues. If associated with organ dysfunction, it is called sepsis and is characterized by a high mortality. ${ }^{1}$

The process known as systemic inflammatory response syndrome (SIRS) (Table 2) does not imply a lack of regulation or organ dysfunction itself. ${ }^{1,22}$ In addition, it may be present in clinical situations not related to an infection (pancreatitis, major burns, multiple trauma, etc.).

- Body temperature elevation through direct modification of the hypothalamic set-point.

- Hypotension due to a vascular (vasodilation) and direct cardiodepressive effect.

- Elevated acute phase proteins (CRP, MBL, $\alpha-2$ macroglobulin, etc.), mediated by direct action on the hepatocyte.

- Coagulation activation; leukocytosis with neutrophilia.

- Induction of an inflammation status at the vascular endothelium due to increased post-capillary venule permeability and increased adhesion molecule expression.

- Increased microbicidal capacity of neutrophil granulocytes and macrophages.

- Macrophage self-induced activation and/or strengthening of induced activation by PAMPs and DAMPs.

- Increased class I HLA molecule expression and increased CD8+ T cells cytotoxic activity.

- Increased stress-related hormone release. Direct stimulation of lipolysis and gluconeogenesis.

PAMPs: pathogen-associated molecular patterns; DAMPs: damage-associated molecular patterns; CRP: C-reactive protein; MBL: mannose-binding lectin; HLA: human leukocyte antigen. 
FIGURE 3. Simplified chart of complement activation pathways

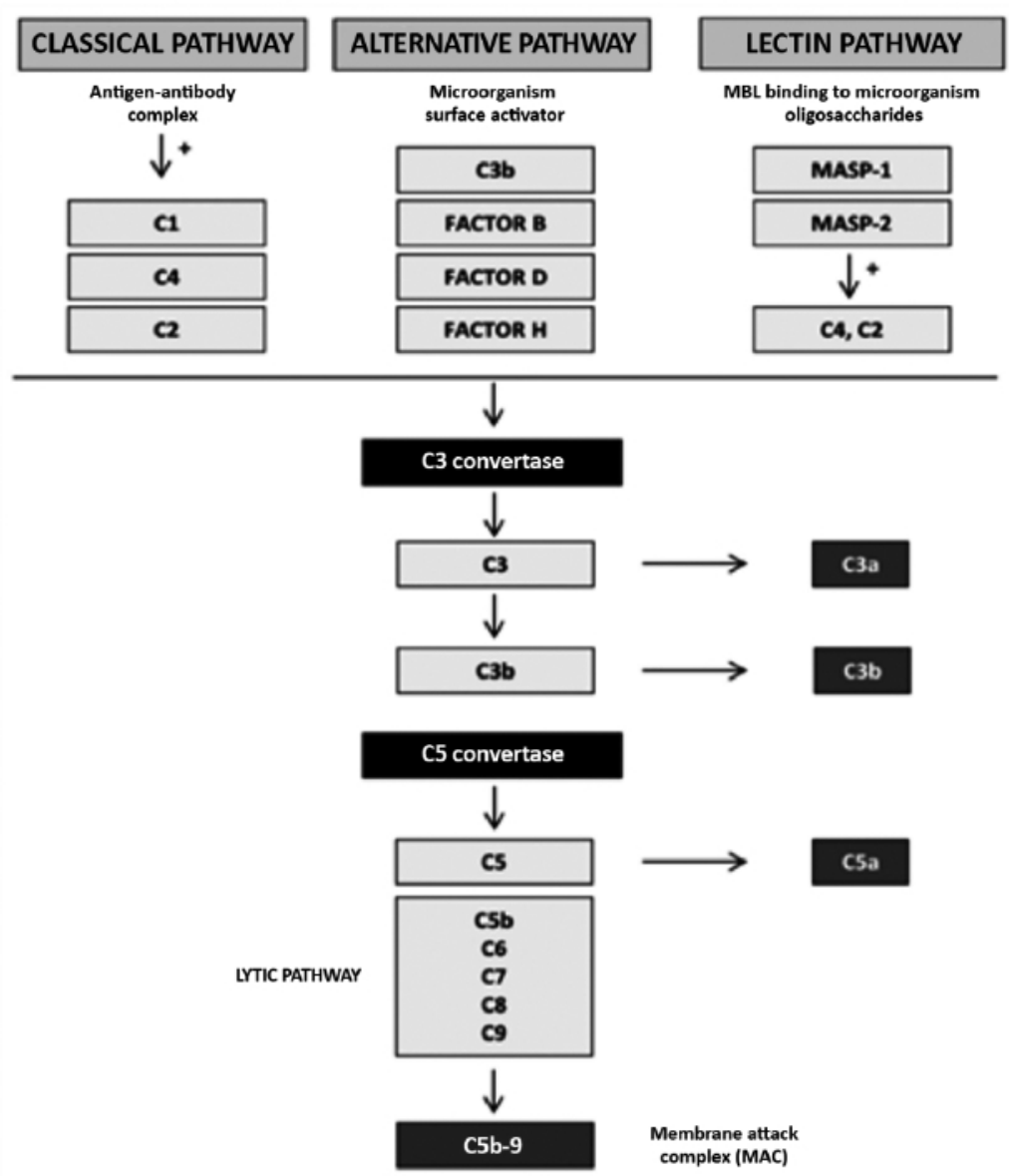

MBL: mannose-binding lectin.

Source: Developed by the author.

TABLE 2. Heart rate, respiratory rate, and white blood cell count values to define systemic inflammatory response syndrome in pediatrics

\begin{tabular}{lccc}
\hline Age & HR & RR & WBC count \\
\hline $0-7$ días & $>180 \mathrm{o}<100$ & $>50$ & $>34000$ \\
$0-7$ days & $>180$ or $<100$ & $>50$ & $>34000$ \\
7 days-1 month & $>180$ or $<100$ & $>40$ & $>19500$ or $<5000$ \\
1 month-1 year & $>180$ or $<90$ & $>34$ & $>17500$ or $<5000$ \\
$>1$ year-5 years & $>140$ & $>22$ & $>15500$ or $<6000$ \\
$>5$ years-12 years & $>130$ & $>18$ & $>13500$ or $<4500$ \\
$>12$ years-18 years & $>110$ & $>14$ & $>11000$ or $<4500$ \\
\hline
\end{tabular}

HR: heart rate in beats per minute; RR: respiratory rate in breaths per minute; WBC: while blood cells per microliter.

Systemic inflammatory response syndrome is defined as 2 or more of the following criteria, at least one of which should be body temperature or WBC count:

- Core temperature $>38.5^{\circ} \mathrm{C}$ or $<36^{\circ} \mathrm{C}$.

- HR $>2$ SD for each age group or $<2$ SD in infants younger than 1 year old.

- $R R>2$ SD for each age group or assisted mechanical ventilation requirement.

- Increased or reduced WBC count for age or $>10 \%$ of immature forms.

Source: modified from Goldstein B, Giroir B, Randolph A, et al. International pediatric sepsis consensus conference: Definitions for sepsis and organ dysfunction in pediatrics. Pediatr Crit Care Med. 2005;6:2. 


\section{PROGRESSION TO SEPSIS}

This process depends on an immense number of events; many of them, related to the microorganism or the host, have not been fully elucidated yet. It is the result of an imbalance in favor of pro-inflammatory phenomena where the inflammatory response exceeds the local infection boundaries and escapes its usual homeostatic regulation, especially in genetically predisposed individuals. ${ }^{22}$

\section{Host factors}

Age, environment, and immune status are decisive factors in progression to sepsis. ${ }^{2,3}$ The production of high pro-inflammatory and antiinflammatory cytokine levels is related to sepsis development. ${ }^{15,16}$ The kinetic analysis shows that TNF- $\alpha$, IL-10, and IL-6 plasma levels reach their peak in the initial process stages, whereas they decrease to almost negligible levels towards the end ${ }^{23}$ (Figure 4. A). These cytokines produce the typical effects of sepsis, including fever, hypotension, endothelial activation, other proinflammatory cytokine release, and coagulation activation. The symptoms produced by TNF- $\alpha$ infusion in animals are similar to those observed during septic shock, ${ }^{14}$ and the experimental infusion of anti-TNF- $\alpha$ antibodies reduced mortality in animals after a lipopolysaccharide challenge. ${ }^{16,17}$ Pro-inflammatory cytokines also inhibit activated macrophage and neutrophil apoptosis, thus extending their survival and increasing inflammatory response. They also induce lymphocyte, dendritic cell, and endothelial cell apoptosis. ${ }^{24}$

The complement plays a major role too. In experimental sepsis models, the inhibition of several of its activated components has allowed to reduce mortality and stop inflammation. . $1,25-28^{2}$ There is also certain genetic susceptibility to sepsis. The most common genetic variation, single nucleotide polymorphism (SNP), was studied as a predictor of progression to sepsis. ${ }^{29}$ SNPs are stable single base substitutions distributed throughout the genome, which includes gene promoters and regions between genes. Many SNPs are related to a higher susceptibility to infections and poorer outcomes in the course of an acute infection..$^{29}$ These SNPs include genes coding structures related to inflammatory response: cytokines (TNF- $\alpha$, IL-1, IL-6, lymphotoxin- $\alpha$, IL-10, IL-18, etc.), interferon $\gamma($ IFN- $\gamma)$, cell surface receptors (TLR-2, TLR-4 and Fc- $\gamma$ II and III receptors), lipopolysaccharide ligand, MBL,
HSP-70, angiotensin-converting enzyme-1, and tissue plasminogen activator inhibitor-1.

\section{Microorganism factors}

Microorganisms play a relevant role in progression to sepsis. Lipopolysaccharides are responsible for triggering the inflammatory cascade in Gram-negative bacterial infections, whereas peptidoglycans, muramyl dipeptides, and lipoteichoic acid do so in Gram-positive bacterial infections.

Lipopolysaccharide biological activity is mediated by an acute phase glycoprotein called lipopolysaccharide-binding protein (LBP). LBPs form a complex with the lipopolysaccharides, which facilitate their interaction with TLR-4 in inflammatory cells and vascular endothelium. The importance of such interaction is evidenced in the lower sensitivity to lipopolysaccharide effects in LBP gene-deficient animals. ${ }^{30}$

When lipopolysaccharides are infused to human beings, they can reproduce many of the clinical characteristics of sepsis. Besides activating the chain of events leading to systemic inflammation, lipopolysaccharides are potent activators of coagulation through an extrinsic (tissue factor expression) and intrinsic (activation of contact phase proteins) pathway. Together with complement activation and an imbalance between coagulation and fibrinolysis, lipopolysaccharides lead to the development of microvascular thrombosis. ${ }^{31,32}$ High circulating lipopolysaccharide levels are associated with a higher risk for progression to septic shock and multiple organ dysfunction. ${ }^{33}$

Other bacterial products (staphylococcal enterotoxin B, toxic shock syndrome toxin-1, Pseudomonas exotoxin A, M protein of group A beta-hemolytic streptococci), as well as changes in microorganism structure (membrane switching into the L-form, greater capsular polysaccharide synthesis, biofilm formation, etc.) also favor sepsis development. ${ }^{34,35}$

\section{BIOLOGICAL MARKERS OF SEPSIS}

Sepsis may be clinically confounded with other entities causing organ dysfunction. To date, more than 170 biological markers have been studied; none of them was sensitive and specific enough to confirm sepsis diagnosis. ${ }^{36} \mathrm{WBC}$ count and some acute phase proteins, including $\mathrm{C}$-reactive protein (CRP), are usually used in spite of their limitations. ${ }^{37}$ The diagnostic performance of procalcitonin is better than that of other sepsis 
biological markers. ${ }^{37,38}$ The measurement of pro-inflammatory cytokines (Figure 4. A) is not usually available. In addition, they may increase with similar kinetics in viral infections and autoinflammatory processes. ${ }^{39,40}$

\section{Acute phase proteins}

CRP, MBL, and other acute phase proteins (Table 3) are synthesized by hepatocytes in response to pro-inflammatory cytokines (IL-1, IL-6, TNF- $\alpha$ ), whereas albumin ceases to be synthesized. CRP is able to recognize exposed phosphorylcholine groups on microorganism surface. This allows its collagen-type domain to bind to and activate $\mathrm{C} 1 \mathrm{q}$, which leads to the activation of the complement classical pathway (Figure 3). In turn, it is capable of opsonizing several microorganisms. ${ }^{41,42}$ On its side, MBL binds to mannose residues exposed on the surface of various microorganisms and mediates two biological actions: it activates complement $\mathrm{C} 4$ and $\mathrm{C} 2$ which trigger the lectin pathway (Figure 3) and opsonizes pathogenic microorganisms. ${ }^{43}$

In general, circulating CRP increases 6 hours after infectious aggression and reaches its peak between 24 and 48 hours (Figure 4. B). This phenomenon also takes place in viral infections (with a lower peak) and autoinflammatory conditions. ${ }^{44}$ The half-life of CRP is constant (between 4 and 9 hours), so circulating levels are directly related to its production rate (inflammatory activity). ${ }^{42}$ Erythrocyte sedimentation rate increases more slowly than CRP (as well as its return to normality) because it is mainly related to fibrinogen production and its circulating half-life ${ }^{45}$ (over 100 hours).

\section{Procalcitonin}

This is a 114-amino acid molecule with no hormonal activity. To date, it is known to interfere in inflammatory response modulation and induce nitric oxide production by the vascular endothelium. In healthy individuals, it cannot be detected in the circulation because it is not released in the absence of systemic inflammation. ${ }^{38}$ In the case of bacterial sepsis, procalcitonin synthesis is activated in almost all body tissues, induced by the direct action of lipopolysaccharides and the effect of several cytokines (TNF- $\alpha$, IL-1, IL-6). Its production is attenuated by IFN- $\gamma$, a cytokine released in response to viral infections; for this reason, procalcitonin is specific for bacterial infections. ${ }^{46}$ Procalcitonin levels start increasing 4-6 hours after the infectious stimulus and reach their peak between 12 and 36 hours (Figure 4. C). Procalcitonin has a diagnostic value because elevated levels are highly suggestive of sepsis $(\geq 2 \mathrm{ng} / \mathrm{mL}),{ }^{38}$ as well as a prognostic value because a reduced circulating level has a very adequate correlation to antibiotic therapy response..$^{37,47}$

FIGURE 4. Kinetics of several inflammatory response mediators after challenge with lipopolysaccharide in human volunteers

A

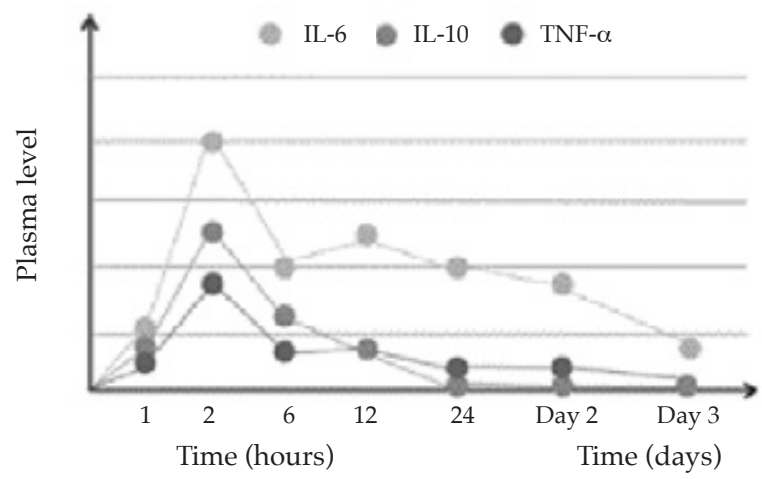

B

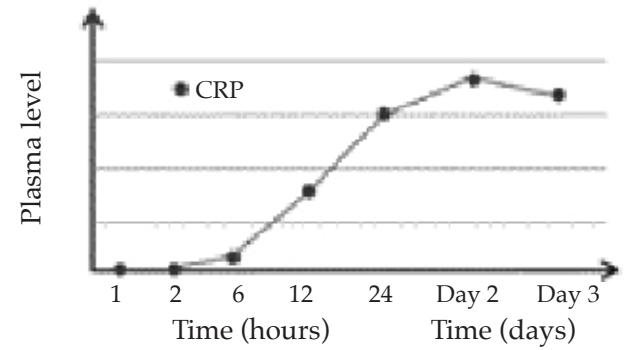

C

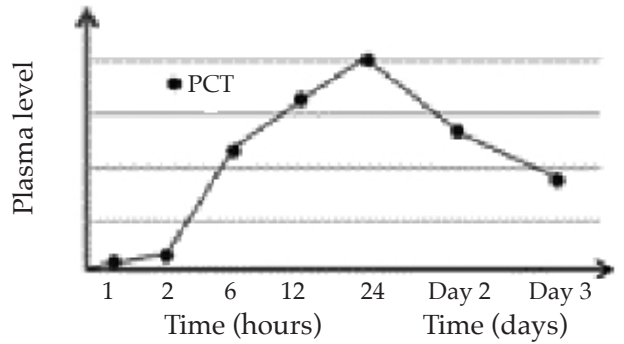

IL-6: interleukin 6; IL-10: interleukin 10; TNF- $\alpha$ : tumor necrosis factor alpha; CRP: C-reactive protein; PCT: procalcitonin. Source: modified from Brunkhorst FM, Heinz U, Forycki ZF. Kinetics of procalcitonin in iatrogenic sepsis. Intensive Care Med. 1998 Aug;24(8):888-9. 
TABLE 3. Main acute phase proteins and their biological function

\begin{tabular}{ll}
\hline Acute phase proteins & Biological function \\
\hline CRP & Complement activation. Pathogen opsonization. \\
MBL & Complement activation. Pathogen opsonization. \\
$\alpha 2$-macroglobulin & Hemostasis modulation. \\
Ferritin & Iron storage. Reduced bioavailability. \\
Hepcidin & Ferroportin-1 internalization. Reduced iron bioavailability. \\
Ceruloplasmin & Iron oxidation. Iron binding to ferritin molecule. \\
Haptoglobin & Hemoglobin transport. Iron binding inside the heme group. \\
Fibrinogen, prothrombin, factor VIII, & \\
Von Willebrand factor & Hemostasis and tissue repair. Inflammatory cell chemotaxis. \\
Plasminogen & Fibrin clot dissolution and tissue repair. \\
\hline
\end{tabular}

CRP: C-reactive protein; MBL: mannose-binding lectin.

\section{CONCLUSION}

Knowledge about the complex mechanisms involved in systemic inflammation and sepsis is becoming increasingly more accurate. Its clinical usefulness lies in a search for biological markers, together with an early diagnosis, that allow to differentiate it from similar clinical entities (hemophagocytic lymphohistiocytosis, adrenal insufficiency, anaphylaxis, etc.). In addition, it is possible to look for new therapeutic targets capable of reducing morbidity and mortality.

\section{REFERENCES}

1. Singer M, Deutschman C, Seymour CW,Shankar-HariM, et al. The Third International Consensus Definitions for Sepsis and Septic Shock (Sepsis-3). JAMA. 2016; 315(8):801-10.

2. Watson RS, Carcillo JA, Linde-Zwible WT, Clermont G, et al. The epidemiology of severe sepsis in children in the United States. Am J Respir Crit Care Med. 2003; 167(5):695701.

3. De Souza DC, Machado FR. Epidemiology of Pediatric Septic Shock. J Pediatr Intensive Care. 2019; 8(1):3-10.

4. Cinel I, Opal SM. Molecular biology of inflammation and sepsis: a primer. Crit Care Med. 2009; 37(1):291-304.

5. Bateman S, Seed P. Procession to Pediatric Bacteremia and Sepsis: Covert Operations and Failures in Diplomacy. Pediatrics. 2010; 126(1):137-50.

6. Bone RC. Immunologic dissonance: a continuing evolution in our understanding of the systemicinflammatory response syndrome (SIRS) and the multiple organ dysfunction syndrome (MODS). Ann Intern Med. 1996; 125(8):680-7.

7. Clapp DW. Developmental regulation of the immune system. Semin Perinatol. 2006; 30(2):69-72.

8. Rittirsch D, Flierl MA, Ward PA. Harmful molecular mechanisms in sepsis. Nat Rev Immunol. 2008; 8(10):776-87.

9. Goldstein B, Giroir B, Randolph A. International pediatric sepsis consensus conference: definitions for sepsis and organ dysfunction in pediatrics. Pediatr Crit Care Med. 2005; 6(1):2-8.

10. Van Amersfoort ES, Van Berkel TJ, Kuiper J. Receptors, mediators, and mechanisms involved in bacterial sepsis and septic shock. Clin Microbiol Rev. 2003; 16(3):379-414.

11. DixonDR, Darveau RP. Lipopolysaccharideheterogeneity: innate host response to bacterial modifications of lipid structure. J Dent Res. 2005; 84(7):584-95.

12. Chen GY, Nuñez G. Sterile inflammation: sensing and reacting to damage. Nat Rev Immunol. 2010; 10(12):826-37.

13. Elson G, Dunn-Siegrist I, Daubeuf B, Pugin J. Contribution of Toll-like receptors to the innate immune response to Gram-negative and Gram-positive bacteria. Blood. 2007; 109(4):1574-83.

14. Van der Poll T, Lowry SF. Tumor necrosis factor in sepsis: mediator of multiple organ failure or essential part of host defense? Shock. 1995; 3(1):1-12.

15. Pinsky MR, Vincent JL, Deviere J, Alegre M, et al. Serum cytokine levels in human septic shock. Relation to multiplesystem organ failure and mortality. Chest. 1993; 103(2): 565- 75.

16. Pruitt JH, Copeland EM $3^{\text {rd }}$, Moldawer LL. Interleukin-1 and Interleukin-1 antagonism in sepsis, systemic inflammatory response syndrome, and septic shock. Shock. 1995; 3(4):235-51.

17. Fresno M, Kopf M, Rivas L. Cytokines and infectious diseases. Immunol Today. 1997; 18(2):56-8.

18. Walport MJ. Complement. First of two parts. N Engl J Med. 2001; 344(14):1058-66.

19. Walport MJ. Complement. Second of two parts. N Engl J Med. 2001; 344(15):1140-4.

20. Monk PN, Scola AM, Madala P, Fairlie DP. Function, structure and therapeutic potential of complement C5a receptors. Br J Pharmacol. 2007; 152(4):429-48.

21. Riedemann NC, Guo RF, Neff TA, Laudes IJ, etal. Increased C5a receptor expression in sepsis. J Clin Invest. 2002; 110(1):101-8.

22. Kawasaki T. Update on pediatric sepsis: a review. Jntensive Care. 2017; 5:47.

23. Brunkhorst FM, Heinz U, Forycki ZF. Kinetics of procalcitonin in iatrogenic sepsis. Intensive Care Med. 1998; 24(8):888-9.

24. Hotchkiss RS, Swanson PE, Freeman BD, Tinsley KW, et al. Apoptotic cell death in patients with sepsis, shock, and multiple organ dysfunction. Crit Care Med. 1999; 27(7):1230-51.

25. Furebring M, Hakansson LD, Venge P, Nilsson B, et al. Expression of the C5a receptor (CD88) on granulocytes and monocytes in patients with severe sepsis. Crit Care. 2002; 6(4):363-70.

26. Huber-Lang MS, Younkin EM, Sarma JV, McGuire SR, et al. Complement-induced impairment of innate immunity 
during sepsis. J Immunol. 2002; 169(6):3223-31.

27. Liu D, Lu F, Qin G, Fernandes SM, et al. C1 inhibitormediated protection from sepsis. J Immunol. 2007; 179(6):3966-72.

28. Liu D, Cai S, Gu X, Scafidi J, et al. C1 inhibitor prevents endotoxin shock via direct interaction with lipopolysaccharide. J Immunol. 2003; 171(5):2594-601.

29. Giamarellos-Bourboulis EJ, Opal SM. The role of genetics and antibodies in sepsis. Ann Transl Med. 2016; 4(17):328.

30. Lamping N, Dettmer R, Schröder NW, Pfiel D, et al. LPSbinding protein protects mice from septic shock caused by LPS or gram-negative bacteria. J Clin Invest. 1998; 101(10):2065-71.

31. Suffredini AF, Fromm RE, Parker MM, Brenner M, et al. The cardiovascular response of normal humans to the administration of endotoxin. N Engl J Med. 1989; 321(5): 280-7.

32. Tapper H, Herwald H. Modulation of hemostatic mechanisms in bacterial infectious diseases. Blood. 2000; 96(7):2329-37.

33. Marshall JC, Foster D, Vincent JL, Cook D, et al. Diagnostic and prognostic implications of endotoxemia in critical illness: results of the MEDIC study. J Infect Dis. 2004; 190(3):527-34.

34. Minasyan H. Sepsis: mechanisms of bacterial injury to the patient. Scand J Trauma Resusc Emerg Med. 2019; 27(1):19.

35. Minasyan H. Sepsis and septic shock: Pathogenesis and treatment perspectives. J Crit Care. 2017; 40:229-42.

36. Vincent JL. The clinical challenge of sepsis identification and monitoring. PLoS Med. 2016; 13(5):e1002022.

37. Sager R, Kutz A, Mueller B, Schuetz P. Procalcitonin-guided diagnosis and antibiotic stewardship revisited. BMC Med. 2017; 15(1):15.
38. Gregoriano C, Heilmann E, Molitor A, Schuetz P. Role of procalcitonin use in the management of sepsis. $J$ Thorac Dis. 2020; 12(Suppl 1):S5-15.

39. Beltra JC, Decaluwe H. Cytokines and persistent viral infections. Cytokine. 2016; 82:4-15.

40. Degré $M$. Interferons and other cytokines in bacterial infections. J Interferon Cytokine Res. 1996; 16(6):417-26.

41. Black S, Kushner I, Samols D. C-reactive Protein. J Biol Chem. 2004; 279(47):48487-90.

42. Marnell L, Mold C, Du Clos TW.C-reactive protein:ligands, receptors and role in inflammation. Clin Immunol. 2005; 117(2):104-11.

43. Alagarasu K, Selvaraj P, Swaminathan S, Raghavan S, et al. Mannose binding lectin gene variants and susceptibility to tuberculosis in HIV-1 infected patients of South India. Tuberculosis (Edinb). 2007; 87(6):535-43.

44. Wacker C, Prkno A, Brunkhorst FM, Schlattmann P. Procalcitonin as a diagnostic marker for sepsis: a systematic review and meta-analysis. Lancet Infect Dis. 2013; 13(5): 426-35.

45. Ramsay ES, Lerman MA. How to use the erythrocyte sedimentation rate in paediatrics. Arch Dis Child Educ Pract Ed. 2015; 100(1):30-6.

46. Schuetz P, Albrich W, Mueller B. Procalcitonin for diagnosis of infection and guide to antibiotic decisions: past, present and future. BMC Med. 2011; 9:107.

47. Wirz Y, Meier M, Bouadma L, Luyt C, et al. Effect of procalcitonin-guided antibiotic treatment on clinical outcomes in intensive care unit patients with infection and sepsis patients: a patient-level meta-analysis of randomized Trials. Crit Care. 2018; 22(1):191. 